\title{
COVID-19 and pandemic perinatal mental health in Australia
}

Christopher Polchleb, Lydia Sung

THE PERINATAL PERIOD increases the vulnerability for the development of anxiety and depression, resulting in poorer maternal, neonatal and infant outcomes. ${ }^{1}$ There is evidence of increased adverse perinatal mental health outcomes following natural disasters and stressful life events. ${ }^{2}$ Similarly, the COVID-19 pandemic has been associated with negative mental health outcomes, with a demonstrated increase in anxiety and depressive symptoms among pregnant patients. ${ }^{3}$

One in five women in Australia is not screened antenatally and postnatally for depression and anxiety. ${ }^{4}$ Changes in perinatal healthcare services due to COVID-19 have resulted in an altered antenatal and postnatal schedule, often delivered by telephone appointments, which may further reduce screening, detection and treatment of mental illness.

The Australian COVID-19 response has been remarkably successful in containing its spread, partly owing to social confinement measures, strict quarantine and geographic isolation. These have caused a sense of isolation and loneliness. ${ }^{5}$ State and international border closures, reticence to enlist assistance of older family members due to fears of COVID-19 exposure and reduced perinatal health attendance have increased the sense of unsatisfactory social support, a strong predictor for perinatal mental illness. ${ }^{6}$ Domestic violence, one of the strongest predictors of perinatal mental illness, ${ }^{7}$ increases in times of disaster, and this has been the case in Australia during the pandemic. ${ }^{8}$ Those with a history of mental health problems or drug and alcohol abuse are also at high risk, with restrictions reducing clinical support services.

Between March and October 2020, the number of new callers to the Perinatal Anxiety and Depression Australia (PANDA) helpline doubled, as did call times, suggesting increased frequency and intensity of illness. Most of these calls were from Victoria, which experienced a prolonged lockdown in 2020.

The Australian Government's response to increasing perinatal mental illness has been swift. An additional $\$ 13.6$ million has been granted as a part of the Perinatal Mental Health and Wellbeing Program to extend vital national perinatal mental health services provided by the PANDA, Red Nose and Sands helplines. Additional funding has also been allocated to curb risk factors correlated with poorer mental health, including domestic violence support. Federal funding of telehealth consults has helped reduce barriers to accessing services for parents during the perinatal period. Beyond this, additional COVID-19-specific perinatal mental health initiatives directed at primary care are minimal.

The Australian pandemic experience to date has been different to that of other countries as there has been minimal infection in the community. However, recovery from the psychological stress of living through a pandemic may take years. ${ }^{9}$ Vigilance for perinatal mental health screening among healthcare providers should be promoted during and after the peak of the pandemic. Measures proven to increase screening include education on specific screening techniques and automated reminders to screen. ${ }^{10}$ State health authorities in Australia have made 'exploring COVID-related concerns' a part of their standard perinatal mental health screening algorithm, helping to identify COVID-19-related factors that may contribute to poorer mental health. ${ }^{11}$

Independent of social restriction due to pandemic public health measures and their impact on face-to-face health services, the therapeutic connection with healthcare providers during the perinatal period should be maintained. Thanks to Medicare Benefits Schedule reforms and support from hospitals, the antenatal schedule, mental health counselling and maternal child health services have been delivered via telehealth, a proven format of healthcare for the perinatal population. ${ }^{12}$ Many organisations have chosen to continue telehealth consults 
'post-lockdown'; however, this must not be merely a cost-saving exercise, and patient satisfaction must be prioritised.

In addition to standard clinical practice, general practitioners (GPs) are encouraged to provide COVID-19specific psychological care to reduce the impacts of the pandemic on perinatal mental health. This includes providing accurate information about the COVID-19 pandemic, validating concerns related to the pandemic, promoting self-help and e-mental health resources and actively encouraging positive coping strategies such as daily routine. ${ }^{13}$ Results from current international research will aim to guide identifying risk and protective factors that predict perinatal mental health outcomes related to the pandemic. ${ }^{14}$

The longer-term psychological impacts of the pandemic on perinatal patients and their families are unknown. Pandemic perinatal stressors remain current while 'circuit breaker' lockdowns are still used, international borders remain closed and insufficient evidence to recommend routine COVID-19 vaccination during pregnancy exists. ${ }^{15} \mathrm{GPs}$, maternal child health nurses and midwives should remain vigilant to discuss and screen for COVID-19-related perinatal mental health difficulties.

\section{First published 12 May 2021.}

\section{Authors}

Christopher Polchleb MBBS, FRACGP, DRANZCOG West Gippsland Healthcare Group, Vic

Lydia Sung DPsych (Clin \& ClinNeuro), BA

(Psychology) (Hons), BComm, AMusA, MAPS, FCCN

Clinical Psychologist, Mums Matter Psychology, Vic;

Clinical \& Clinical Neuropsychologist, Austin Child Inpatient Unit - Senior CYMHS Clinician, Vic

Competing interests: None.

Funding: None.

Provenance and peer review: Not commissioned, peer reviewed.

Citation: Polchleb C, Sung L. COVID-19 and pandemic perinatal mental health in Australia. Aust J Gen Pract 2021;50 Suppl 49. doi: 10.31128/AJGPCOVID-49.

Correspondence to:

cpolchleb@gmail.com

\section{References}

1. Grigoriadis S, VonderPorten EH, Mamisashvili L, et al. The impact of maternal depression during pregnancy on perinatal outcomes. The J Clin Psychiatry 2013;74(4):e321-e41. doi: 10.4088/ JCP.12r07968
2. Harville $E$, Xiong $X$, Buekens P. Disasters and perinatal health: A systematic review. Obstet Gynecol Surv 2010;65(11):713-28. doi: 10.1097/ OGX.0b013e31820eddbe.

3. Farrell T, Reagu S, Mohan S, et al. The impact of the COVID-19 pandemic on the perinatal mental health of women. J Perinat Med 2020;48(9):971-76. doi: 10.1515/jpm-2020-0415

4. Moss KM, Reilly N, Dobson AJ, Loxton D, Tooth L, Mishra GD. How rates of perinatal mental health screening in Australia have changed over time and which women are missing out. Aust N Z J Public Health 2020:44(4):301-06. doi: 10.1111/17536405.12999 .

5. Tull MT, Edmonds KA, Scamaldo KM, Richmond JR, Rose JP, Gratz KL. Psychological outcomes associated with stay-at-home orders and the perceived impact of COVID-19 on daily life. Psychiatry Res 2020;289:113098. doi: 10.1016/j.psychres.2020.113098

6. Boyce P, Hickey A. Psychosocial risk factors to major depression after childbirth. Soc Psychiatry Psychiatr Epidemiol 2005;40(8):605-12. doi: 10.1007/s00127-005-0931-0.

7. Lancaster CA, Gold KJ, Flynn HA, et al. Risk factors for depressive symptoms during pregnancy: A systematic review. Am J Obstet Gynecol 2010;202(1):5-14. doi: 10.1016/j. ajog.2009.09.007

8. Neil J. Domestic violence and COVID-19: Our hidden epidemic. Aust J Gen Pract 2020;49 Suppl 25. doi: 10.31128/AJGP-COVID-25.

9. Smith GP, Wenger D. Sustainable disaster recovery: Operationalizing an existing agenda. In: Rodriguez H, Quarantelli E, Dynes R, editors. Handbook of disaster research. New York, NY: Springer Science + Business Media, 2006; p. 234-57.

10. Long MM, Cramer RJ, Jenkins J, Bennington L, Paulson JF. A systematic review of interventions for healthcare professionals to improve screening and referral for perinatal mood and anxiety disorders. Arch Womens Ment Health 2019;22(1):25-36 doi: 10.1007/s00737-018-0876-4

11. Queensland Centre of Perinatal and Infant Mental Health. Perinatal mental health screening in COVID-19: Clinical guidance note. Nundah, Qld: QCPIMH, 2020

12. Implementing telehealth in practice. Obstet Gynecol 2020;135(2):e73-e9. doi: 10.1097/ AOG.0000000000003671.

13. Motrico E, Mateus V, Bina R, et al. Good practices in perinatal mental health during the COVID-19 pandemic: A report from task-force RISEUP-PPD COVID-19. Clínica y Salud 2020;31(3):155-60. doi:10.5093/clysa2020a26.

14. Motrico E, Bina R, Domínguez-Salas S, et al. Impact of the Covid-19 pandemic on perinatal mental health (Riseup-PPD-COVID-19): Protocol for an international prospective cohort study. BMC Public Health 2021;21(1):368. doi: 10.1186/s12889021-10330-w.

15. The Royal Australian and New Zealand College of Obstetricians and Gynaecologists. COVID19 vaccination in pregnant and breastfeeding women. East Melbourne, Vic: RANZCOG, 2021. Available at https://ranzcog.edu.au/ statements-guidelines/covid-19-statement/ covid-19-vaccination-information [Accessed 30 April 2021]. 